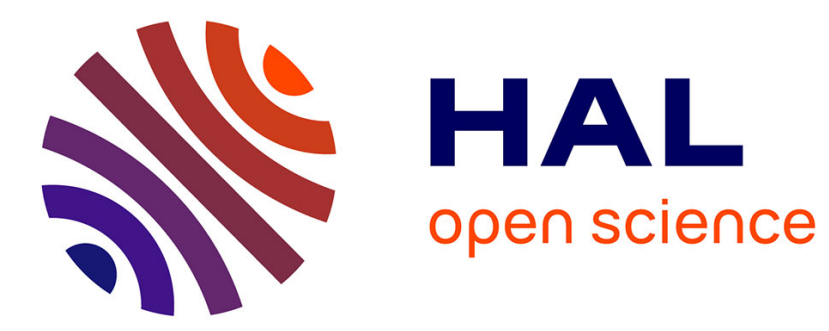

\title{
La fondation de la Banque des règlements internationaux Frédéric Clavert
}

\section{To cite this version:}

Frédéric Clavert. La fondation de la Banque des règlements internationaux. Histoire, économie et société, 2011, 30e année (4), pp.11-17. 10.3917/hes.114.0011 . halshs-01100454

\section{HAL Id: halshs-01100454 \\ https://shs.hal.science/halshs-01100454}

Submitted on 7 Jan 2015

HAL is a multi-disciplinary open access archive for the deposit and dissemination of scientific research documents, whether they are published or not. The documents may come from teaching and research institutions in France or abroad, or from public or private research centers.
L'archive ouverte pluridisciplinaire HAL, est destinée au dépôt et à la diffusion de documents scientifiques de niveau recherche, publiés ou non, émanant des établissements d'enseignement et de recherche français ou étrangers, des laboratoires publics ou privés. 


\title{
LA FONDATION DE LA BANQUE DES RÈGLEMENTS INTERNATIONAUX
}

\author{
Frédéric Clavert
}

Armand Colin | Histoire, économie \& société

\author{
2011/4 - 30e année \\ pages 11 à 17
}

ISSN 0752-5702

Article disponible en ligne à l'adresse:

http://www.cairn.info/revue-histoire-economie-et-societe-2011-4-page-11.htm

Pour citer cet article :

Clavert Frédéric, « La fondation de la Banque des règlements internationaux »,

Histoire, économie \& société, 2011/4 30e année, p. 11-17. DOI : 10.3917/hes.114.0011

Distribution électronique Cairn.info pour Armand Colin.

(c) Armand Colin. Tous droits réservés pour tous pays.

La reproduction ou représentation de cet article, notamment par photocopie, n'est autorisée que dans les limites des conditions générales d'utilisation du site ou, le cas échéant, des conditions générales de la licence souscrite par votre établissement. Toute autre reproduction ou représentation, en tout ou partie, sous quelque forme et de quelque manière que ce soit, est interdite sauf accord préalable et écrit de l'éditeur, en dehors des cas prévus par la législation en vigueur en France. II est précisé que son stockage dans une base de données est également interdit. 


\title{
La fondation de la Banque des règlements internationaux
}

\author{
par Frédéric Clavert
}

\begin{abstract}
Résumé
Cet article s'attache à décrire et à analyser la création de la Banque des Règlements internationaux en 1930. Se fondant essentiellement sur des archives allemandes et françaises, il tente d'expliquer pourquoi les banquiers centraux membres du Comité Young puis du Comité d'organisation de la banque des règlements internationaux ont fini, malgré une attitude ambivalente de la Reichsbank et de Hjalmar Schacht, par se mettre d'accord sur les fonctions et la fondation de la BRI. Dans ce processus, l'accord des banquiers centraux sur l'idée d'indépendance des banques centrales est fondamental.
\end{abstract}

\section{Abstract}

This article aims to describe and analyse the creation of the Bank for International Settlements (BIS) in 1930. Based essentially on German and French archives, it will attempt to explain why the central bankers that were members of the Young Committee and subsequently the Organisation Committee for the Bank for International Settlements (COBRI) eventually reached agreement on the role and founding of the BIS, despite the ambivalent attitude of the Reichsbank and Hjalmar Schacht. In this process, the agreement of the central bankers on the idea of independence of the central banks was fundamental.

L'entre-deux-guerres est une étape importante dans la structuration du « petit peuple des banquiers centraux », notamment par la fondation de la Banque des règlements internationaux ${ }^{1}$. Si cette dernière a été créée en 1930 elle est, finalement, la conséquence de la situation financière internationale issue de la Première Guerre mondiale. En effet, non seulement, en 1919, l'endettement international a atteint des sommets inédits, mais de nouveaux États ou États successeurs de l'Autriche-Hongrie ou de la Russie doivent créer leur propre monnaie, souvent grâce à l'aide des vainqueurs et de leurs banques centrales. En outre, certains pays n'ont pas encore de système monétaire fondé sur une banque centrale à proprement parler ou doivent le réformer. Par exemple, la Hongrie crée sa banque centrale

1. Nous avons consulté pour cet article des archives de la Banque de France, de la Reichsbank et de la Banque des règlements internationaux. 
en 1924 et la Grèce ne le fait qu'en 1927. Ce n'est alors pas une situation exceptionnelle : pour sortir du continent européen, les États Unis n'ont créé la Réserve fédérale qu'en 1913. Ainsi, existe-t-il dans l'entre-deux-guerres un besoin particulier concernant les problèmes monétaires et les banques centrales elles-mêmes.

Pourtant, dans les années 1920 et 1930, les conflits entre banques centrales et entre leurs gouverneurs sont nombreux. Si l'on prend l'exemple du Comité des experts de Paris en 1929 - qui a permis la mise en place du plan Young en remplacement du plan Dawes de 1924 et la création de la BRI - les notes de ses membres évoquent une série, parfois tragi-comique, de rebondissements liés à des heurts entre banquiers centraux, opposant notamment Émile Moreau, gouverneur de la Banque de France, et Hjalmar Schacht, président de la Reichsbank, sur les annuités que doit payer l'Allemagne ${ }^{2}$. Schacht s'est également souvent confronté à Émile Franqui, délégué belge, sur les marks belges ou le choix du siège de la Banque des Règlements internationaux ${ }^{3}$. Enfin, le jeu trouble de Montagu Norman, parfois en opposition à la position officielle du Trésor britannique, accentue ces dissensions, même si Norman n'est pas membre du Comité ${ }^{4}$. Au vu de ces divisions, pourquoi les banquiers centraux ont-ils proposé et accepté la création de la BRI ?

\section{L'idée de banque internationale dans les années 1920}

À partir du début des années 1920, notamment autour de Montagu Norman, les notions de banques centrales et banquiers centraux sont progressivement mieux définies. Deux points ressortent particulièrement : la notion d'indépendance de la banque centrale vis-à-vis de l'État d'une part ; les relations exclusives entre les banques centrales d'autre part. Ces deux idées fondamentales mettent globalement d'accord les banquiers centraux ${ }^{5}$.

Cette définition du rôle des banquiers centraux croise une autre tendance : la nécessité d'une institution financière internationale, qui émerge dès la conférence de Paris à l'issue de la guerre. En 1929, l'idée d'une banque internationale n'est en effet plus originale : dès avril 1919, Lloyd George reprend une idée de Keynes d'un emprunt pour l'Europe centrale et orientale, qui comprend la création d'une institution financière pour gérer cet emprunt ${ }^{6}$. Le but des Britanniques est alors de stabiliser cette région et d'assurer le paiement des réparations d'une part et la fluidité des paiements internationaux malgré le poids que représentent les réparations d'autre part.

2. Voir, pour le détail de ce conflit Moreau/Schacht : Frédéric Clavert, « Reichsbank et Banque de France face aux négociations sur le plan Young », Revue d'Allemagne et des pays de langue allemande, 2007, vol. 39, $\mathrm{n}^{\mathrm{o}} 2$.

3. Voir, pour un panorama des relations de Schacht avec la Belgique : Frédéric Clavert, « Hjalmar Schacht et la Belgique », in Birte Wassenberg et Martial Libera (dir.), L'Europe au cour. Livre offert à Marie-Thérèse Bitsch, Bruxelles, PIE - Peter Lang, 2009.

4. C'est particulièrement le cas lors de la préparation des travaux du comité, pendant la seconde moitié de 1928. Voir Frédéric Clavert, Hjalmar Schacht, financier et diplomate : 1930-1950, Bruxelles, PIE - Peter Lang, 2009, p. 57.

5. Sur ce sujet, voir notamment: Olivier Feiertag, «Banques centrales et relations internationale au $\mathrm{XX}^{\mathrm{e}}$ siècle : le problème historique de la coopération monétaire internationale », Relations internationales, 1999, $\mathrm{n}^{\circ}$ 100, p. 355-376 ; Olivier Feiertag et Michel Margairaz, L'Internationalisation des banques centrales, Paris, Presses de Sciences po, à paraître, et plus particulièrement la contribution de Philip Cottrell ; Olivier Feiertag et Michel Margairaz, Politiques et pratiques des banques d'émission en Europe (XVII $-X X^{e}$ siècle) : le bicentenaire de la Banque de France dans la perspective de l'identité monétaire européenne, Paris, Albin Michel, 2003, 832 p.

6. Sylvain Schirmann, Quel ordre européen : de Versailles à la chute du III ${ }^{e}$ Reich, Paris, Armand Colin, 2006, p. 37. 
De plus, et parallèlement aux mouvements de réflexion sur l'unité européenne, certains pensent une Europe économiquement organisée : Walter Rathenau, alors ministre allemand des Affaires étrangères, Joseph Caillaux, ministre Français des Finances en 1925, le Hongrois Elemer Hantos ${ }^{7}$. Avec l'organisation économique vient l'organisation monétaire : l'Appel aux Européens adressé à la SDN le 12 mars 1925 - dont Hantos est signataire proclame que la paix européenne repose sur la paix économique et propose ainsi une Union douanière européenne. Cette dernière devient une association, l'UDE, qui se développe et fait des propositions, notamment celle d'une institution financière européenne ${ }^{8}$. L'idée est reprise par le Français Francis Delaisi en 1929, dans un livre dont la préface du financier belge Dany Heinemann insiste sur la notion d'outil de régulation de l'économie européenne comme une institution financière préfigurant une sorte de banque centrale continentale ${ }^{9}$. Ces deux tendances - le rôle des banques centrales et une institution financière européenne - se rencontrent lors du comité des experts à Paris, qui se tient de février à juin 1929.

\section{Le comité des experts à Paris : première phase de la genèse de la BRI (1929)}

Le comité qui se réunit à Paris de février à juin 1929 sous la présidence de l'homme d'affaires américains Owen D. Young rassemble des experts, nommés par leur gouvernement (France, Allemagne, Belgique, Italie, États-Unis, Japon), mais indépendants d'eux. Parmi les experts, on retrouve des banquiers centraux, notamment Hjalmar Schacht de la Reichsbank et Émile Moreau de la Banque de France. Les experts doivent trouver un remplacement au plan Dawes qui régulait le paiement des réparations par l'Allemagne et qui prévoyait son remplacement au bout de cinq ans. À court terme, le plan Young est un échec : dès 1931, le système des réparations est insoutenable et est définitivement abandonné en 1932 à la conférence de Lausanne. Mais à long terme, la plus belle réalisation du plan est la création de la Banque des règlements internationaux. En somme, les experts ont voulu voir au-delà du règlement des réparations et au-delà des réparations elles-mêmes.

Les discussions du comité se déroulent globalement en trois phases ${ }^{10}$. Dans un premier temps, les experts évoquent des problèmes principalement techniques. Dans un second temps, et plus longuement, ils se sont arrêtés sur le cœur des discussions - c'est-à-dire le montant des annuités payables par l'Allemagne à ses créanciers «politiques » au titre des réparations. Cette seconde partie des discussions est marquée par de nombreux rebondissements - dont les deux derniers sont d'une part une attaque contre le Reichsmark qui, d'après Montagu Norman, provient de la Banque de France ${ }^{11}$, et d'autre part le décès d'un représentant britannique, Lord Revelstoke, qui permet à chaque expert de rentrer dans

7. Ibid., p. 81 .

8. Idem.

9. Francis Delaisi, Les Deux Europes, Paris, s.1., 1929.

10. Pour une description précise des négociations sur le plan Young, consulter : Frédéric Clavert, Hjalmar Schacht, financier et diplomate : 1930-1950, op. cit., p. 69-82 ; Frédéric Clavert, « Reichsbank et Banque de France face aux négociations sur le plan Young », op. cit.; Philipp Heyde, Das Ende der Reparationen : Deutschland, Frankreich und der Youngplan 1929-1932, Paderborn, F. Schöningh, 1998 ; André Pepy, Le Plan Young, Paris, Éditions internationales, 1930. Dans notre cas, outre les papiers Schacht à Coblence - Bundesarchiv (BArch) Koblenz, Teilnachlass Schacht, N1294 - et les archives de la Reichsbank - BArch Berlin, Deutsche Reichsbank, R2501-, nous avons consulté les archives de la Banque de France et particulièrement les notes de Clément Moret : Archives de la Banque de France, 1489200303/11, « Documents de réunion de M. Moret ». Nous avons eu également accès aux archives de la BRI, BR07, « Committee of experts ».

11. Akten zur Deutschen Auswärtigen Politik (ADAP), Kabinett Müller II, Band 1, n 190, « Besprechung über die Reparationslage. 1. Mai 1929, 17.30 Uhr ». Néanmoins, pour Robert Boyce, la Banque de France n'est pas intervenue : Robert BOYCE, «La Banque de France et la Banque d'Angleterre dans l'entre-deux-guerres : origines et conséquences d'un conflit », in Olivier Feiertag et Michel Margairaz, Politiques et pratiques des 
son pays et de consulter son gouvernement sur les risques d'échec du comité ${ }^{12}$. Au retour de cette pause forcée, l'Allemagne cède sur le montant des annuités, par peur qu'un échec des discussions n'emporte sa monnaie. S'ouvre alors la dernière phase du comité, lorsque les experts discutent des points techniques liés aux annuités. Le plan des experts est publié au début du mois de juin, après un ultime feuilleton mouvementé autour de la question des «marks belges ${ }^{13}$. Ces derniers sont les marks laissés par l'Allemagne lorsque la Belgique a été évacuée à la fin de la Grande Guerre, mais qui ont perdu toute valeur avec la stabilisation du mark en 1923-1924 ${ }^{14}$.

La banque des règlements internationaux serait une idée de Hjalmar Schacht ${ }^{15}$. En fait, les discussions en 1929 à Paris montrent que cette version des faits est une légende sans fondement ${ }^{16}$. Cette banque est une création commune, reposant sur une idée partagée par les banquiers centraux : la nécessité de coopération entre les instituts d'émission. Le comité des experts s'est divisé en trois commissions sur trois sujets différents. Les trois commissions reviennent avec un rapport des discussions qui s'y sont tenues. Chacune propose la création d'un institut disposant de certaines compétences d'une banque internationale. C'est à ce moment-là que Schacht évoque une clearing house, c'est-à-dire une chambre de compensation, et que les Belges produisent un mémorandum évoquant une banque internationale le 25 février $1929^{17}$. C'est ensuite Owen Young qui promeut cette idée de banque internationale auprès des autres délégations. Ainsi la BRI est-elle une création commune des banquiers centraux ${ }^{18}$.

Quel était le but de cette banque internationale ? La BRI doit métamorphoser les réparations en problème strictement commercial et aider l'Allemagne à accroître ses exportations pour lui permettre de payer plus facilement ses annuités. Cette banque devait ainsi aider le Reich à corriger les structures de son économie et à accroître sa solvabilité. Toutefois, les experts ont voulu en faire un élément central de la coopération entre les banques centrales ${ }^{19}$. Il est prévu qu'elle soit dirigée par les banques centrales elles-mêmes, qui en seraient les actionnaires et qui disposeraient ainsi des droits de vote à l'Assemblée générale et au Conseil d'administration. Ses bénéfices devaient permettre la constitution d'un fonds de réserve, devant éventuellement aider l'Allemagne à payer les annuités. Elle était chargée de placer sur le marché international une partie des réparations, pour permettre une certaine souplesse aux paiements et à leur transfert. Elle pouvait notamment investir en Allemagne, pour dynamiser son économie tout en fournissant les devises nécessaires pour les paiements. Surtout, il est très vite prévu que la BRI utilise ces placements dans l'intérêt du commerce international pour stabiliser les changes

banques d'émission en Europe (XVII $-X X^{e}$ siècle) : le bicentenaire de la Banque de France dans la perspective de l'identité monétaire européenne, op. cit., p. 465.

12. Dans le cas allemand, ces discussions entre experts ont lieu à Berlin, notamment le $1^{\text {er }}$ mai 1929 : ibid.

13. Frédéric Clavert, Hjalmar Schacht, financier et diplomate : 1930-1950, op. cit., p. 75-77.

14. Voir infra.

15. Hjalmar Schacht, 76 Jahre meines Lebens, Kindler und Schiermeyer Verlag, 1953, p. 313.

16. Archives de la BRI, BR07, "Committee of experts, Draft regulations of the "Clearing House" », Schacht's Memo. to Mr Young.

17. Ibid.

18. C'est en tout cas ce qu'il ressort de la lecture des notes prises pendant les sessions du Comité de Clément Moret : Archives de la Banque de France, 1489200303/11 « Documents de réunion de M. Moret ».

19. Ils l'ont exprimé eux-mêmes dans le rapport final - le plan Young - dans sa IV e partie. 
et faciliter les paiements internationaux. Elle obtient également une mission générale d'encouragement à la coopération entre banque centrale ${ }^{20}$.

Cette idée de banque internationale, émise dès la fin du mois de février 1929, n'est plus remise en cause par les experts pendant les sessions du Comité, malgré la brutalité des échanges sur le montant des annuités, sauf lors de la discussion sur les marks belges. Ces derniers avaient été créés, sur la base d'un emprunt des banques belges garanti sur l'or, pour gérer financièrement l'occupation de la Belgique par l'Allemagne pendant la Première Guerre mondiale. Après la défaite du Reich, la Reichsbank a intégré cet emprunt-or dans ces réserves, mais la Belgique garde ses marks. Dans un premier temps, la situation est plutôt avantageuse pour Bruxelles : ces marks facilitent les achats de produits allemands. Mais l'hyperinflation de 1923 et la création du Rentenmark puis du Reichsmark en 1924 changent la donne : ces marks belges perdent toute valeur. La Belgique a ensuite régulièrement essayé d'obtenir indemnisation. En 1926, un entretien entre le Belge Léon Delacroix, ancien Premier ministre belge, et Schacht évoque un paiement de ces marks en échange du retour d'Eupen et Malmédy à l'Allemagne - discussions qui mettent en danger Locarno ${ }^{21}$. Lorsque le comité des experts est convoqué le 16 septembre 1928 et finalement réuni en février 1929, la Belgique annonce son intention de demander aux experts de résoudre cette question, condition sine qua none de son adhésion à un nouveau plan ${ }^{22}$. Or, pour Schacht, un remboursement de ces marks sans contrepartie reviendrait à une sorte de réévaluation d'une petite partie des anciens marks - en clair une remise en cause de la stabilisation de $1923^{23}$. Cependant, de février à juin, il exploite cette situation pour pousser les Belges sans succès - à parler d'Eupen et Malmédy ${ }^{24}$.

Intervient alors une seconde question, celle du siège de la Banque des Règlements internationaux. Bruxelles souhaitait l'accueillir. Schacht s'y oppose et utilise les marks belges et Eupen et Malmédy pour empêcher que le siège soit à Bruxelles : pour le président de la Reichsbank, il est hors de question que la BRI se situe dans un pays en conflit territorial avec le Reich. Franqui et Schacht, finalement, atteignent un niveau de discussion tellement conflictuel que Bruxelles et Berlin retirent du mandat de leurs experts la capacité de discuter des marks belges. L'Allemagne s'engage à régler la question au plus tôt - ce qui est fait le 13 juillet 1929 par un accord intergouvernemental entre les deux pays $^{25}$.

Cette instrumentalisation des marks belges par Schacht est le seul moment, pendant les discussions des experts, où la BRI est remise en cause par un banquier central.

Une fois le plan Young publié, la création de la BRI apparaît clairement comme la pierre angulaire du mémorandum des experts et du paiement des réparations. Elle est évoquée dans sa quatrième partie et son annexe I. La BRI est ainsi justifiée : "Nous estimons que, par un développement financier judicieux et exempt d'esprit de concurrence, la banque constituera un instrument utile pour ouvrir de nouveaux débouchés au commerce

20. Quatrième partie du plan Young, complété par l'annexe I. Le plan Young et ses annexes ont été reproduits dans André Pepy, Le Plan Young, op. cit.

21. Raymond Poidevin et Jacques Bariéty, Les Relations franco-allemandes : 1815-1975, Paris, Armand Colin, 1979, p. 270.

22. Franqui contacte Schacht dès le troisième jour de réunion du comité : ADAP, Kabinett Müller II, Band $1, \mathrm{n}^{\circ} 152$.

23. BArch Koblenz, Nachlass Schacht, N/1294/5, Schacht à Müller, 8 mai 1929.

24. BArch Koblenz, Nachlass Schacht, N/1294/5, Schacht à Franqui, 8 mai 1929.

25. Frédéric Clavert, Hjalmar Schacht, financier et diplomate : 1930-1950, op. cit., p. 77. 
et aux échanges internationaux et contribuera ainsi à résoudre le problème particulier de l'Allemagne sans empiéter sur l'activité des établissements existants ${ }^{26}$. »

Il s'agit d'une coopération volontaire des banques centrales pour faciliter le commerce en facilitant les paiements internationaux. La BRI va ainsi bien au-delà du système de règlement des réparations. Les experts prévoient immédiatement les modalités pratiques de mise en œuvre de la BRI en convoquant un Comité d'Organisation de la Banque des Règlements internationaux (COBRI).

\section{Un accouchement dans la douleur : du COBRI à la réunion de Rome}

Le COBRI est prévu par le plan Young. Ce dernier, au moment de sa présentation le 7 juin 1929, n'est qu'un document signé par des experts et n'engageant pas les gouvernements. S'enclenche alors un processus visant à faire de ce plan d'experts un traité international impliquant les États. Une première conférence à La Haye à l'été 1929 convoque une série de commissions thématiques - sur la Reichsbahn, sur la rédaction du traité, etc. - puis une seconde conférence permet de finaliser le traité. Parmi les commissions convoquées par la conférence de La Haye figure le COBRI. Or les banquiers centraux ont mis un point d'honneur à imposer leur indépendance, estimant que cette commission était prévue par le plan Young et, ainsi, qu'elle est indépendante de la présidence de la Conférence de La Haye, ne pouvant donc être subordonnée aux gouvernements. Moreau et Schacht tombent d'accord et organisent le COBRI pour qu'il devienne une déclaration d'indépendance des banquiers centraux ${ }^{27}$. Henri Jaspar, Premier ministre belge et président de la conférence de La Haye, lorsqu'il indique les conditions de financement, d'hébergement et de réunion du COBRI, se voit rétorquer unanimement que le COBRI est prévu par le plan Young et dépend donc des banques centrales $^{28}$. Ces dernières en assurent le financement et en choisissent elles-mêmes le siège ${ }^{29}$.

Les séances du COBRI permettent de mettre au point les documents juridiques nécessaires à la création de la BRI - notamment les accords de trust et les statuts de la BRI. Ce ne sont cependant pas les sessions d'octobre et novembre 1929 qui décident de cette création : peu de temps après les Belges ${ }^{30}$, les Français quittent le COBRI le 11 novembre $1929^{31}$ : pour les Français, les questions restantes dépendent des gouvernements, c'est-à-dire de la conférence de La Haye qui doit se rouvrir en janvier 1930.

Pendant deux mois, la BRI est alors remise en cause par des questions internes allemandes qui marquent le début de la lente agonie de la République de Weimar. Tout au long des discussions des experts à Paris, puis pendant la conférence de La Haye à l'été 1929 et les mois qui suivent, Schacht devient, progressivement, un opposant au gouvernement de coalition dirigée par le social-démocrate Hermann Müller. Le président de la Reichsbank s'est même brouillé avec celui qui lui a permis d'accéder à la tête de la banque centrale allemande, Gustav Stresemann, mort en septembre 1929. Schacht exprime ses griefs contre la politique du gouvernement allemand vis-à-vis du plan Young dans un mémorandum

26. Rapport des experts (Plan Young), partie IV in André Pepy, Le Plan Young, op. cit., p. 123.

27. Banque de France, DGSE, 1489200303/26, Conférence de La Haye, travaux du comité d'organisation (septembre-octobre 1929), lettre de Moreau à Schacht du 2 septembre 1929.

28. Ibid.

29. Ibid.

30. ADAP, Kabinett Müller II, Band 2, $\mathrm{n}^{\circ} 345$ « Die deutsche Delegation des Organisationskomitees der Bank für internationalen Zahlungsausgleich an den Reichskanzler. Baden-Baden, 9. November 1929 »

31. BArch Koblenz, Nachlaß Schacht, N/1294/3, lettre de Schacht à Norman, 13.11.1929, Baden-Baden. 
publié le 6 décembre $1929^{32}$ et remet en cause la participation de la Reichsbank à la BRI dans une lettre du 31 décembre $1929^{33}$ adressée au président de la conférence de La Haye, Jaspar. Avec l'organisation du COBRI, il y a acte d'indépendance des banques centrales. Mais Schacht, l'un d'entre eux, est quand même capable de remettre en cause cette création pour des questions d'abord nationales.

La seconde conférence de La Haye s'ouvre en janvier 1930. L'Allemagne est fragilisée par l'arrivée de Schacht dans la ville néerlandaise le 13 janvier. Le président de la Reichsbank confirme le refus de participation de la Reichsbank à la BRI, estimant que les discussions politiques ont dévoyé l'esprit du plan Young et n'assurent pas l'égalité de l'Allemagne vis-à-vis de ses partenaires internationaux - notamment en ne garantissant pas la pleine indépendance de la Reichsbank ${ }^{34}$. Le Schacht-Complex ${ }^{35}$ est réglé par la délégation allemande. Les Français exerçant une forte pression sur les Allemands, le gouvernement Müller propose alors à Schacht une loi qui obligerait la Reichsbank à adhérer à la BRI. Schacht accepte : cette loi permettrait de ne pas faire porter la responsabilité de cette participation par Reichsbank, mais la reporte sur le gouvernement et les députés allemands $^{36}$. Cet accord ouvre la voie à la création de la BRI, les 26 et 27 février 1930 à Rome, où Schacht est présent. La BRI voit officiellement le jour le 5 mars 1930. Ses premiers pas se font pour l'essentiel sans Schacht, qui démissionne le 7 mars.

\section{Conclusion}

La BRI est née dans la douleur, à cause de la Reichsbank et de Schacht. Mais elle est née car les désaccords entre les banquiers centraux rencontrent une limite, qui est une définition commune de leurs intérêts et, en premier lieu, l'indépendance des banques centrales vis-à-vis du politique. Cette naissance dans la douleur s'explique par le fait que les banques centrales sont prises entre plusieurs niveaux : les relations internationales, les relations entre les banques centrales et la scène nationale. Or, c'est pour des raisons nationales que Schacht est ambivalent vis-à-vis de la création de la BRI, oscillant entre un certain enthousiasme en février-mars 1929 et une remise en cause en décembre-janvier 1929-1930.

Toujours est-il que la création de la BRI nous semble être un moment particulièrement important de la constitution d'une communauté transnationale des banquiers centraux. Cette dernière, en gestation dans les années 1920, rencontre des limites - illustrées par le comportement de Schacht - qui sont renforcées par la crise économique des années 1930 et, surtout, par les solutions nationales qui constituent les réponses les plus déterminantes à cette crise après l'échec des solutions internationales ${ }^{37}$.

32. ADAP, Kabinett Müller II, Band 2, n 369, « Der ReichsbankPräsident an den Reichskanzler. 5. Dezember 1929 ». Schacht a communiqué au gouvernement le mémorandum la veille de sa publication officielle.

33. BArch Koblenz, Nachlass Schacht, N/1294/3, lettre du directoire de la Reichsbank à M. J.A.M. de Sanchez pour transmission à M. J.E. Reynolds, président du COBRI, 31 décembre 1929.

34. BArch Koblenz, Nachlass Schacht, N/1294/3, Vocke à Schacht, 14 janvier 1930.

35. L'expression est de Müller : ADAP, Kabinett Müller II, Band 2, n 417.

36. ADAP, Kabinett Müller II, Band 2, n 416, « Aufzeichnung Staatssekretär Pünders über die Delegationsbesrepchung in Den Haag am 14. Januar 1930 betr. Deutsche Beteiligung an der BIZ ».

37. Voir sur l'échec des solutions internationales à la crise : Sylvain Schirmann, Crise, coopération économique et financière entre États européens, 1929-1933, Paris, Comité pour l'histoire économique et financière de la France, 2000. 